http://jmscr.igmpublication.org/home/ ISSN (e)-2347-176x ISSN (p) 2455-0450

crossref DOI: https://dx.doi.org/10.18535/jmscr/v7i9.90

\author{
Journal Of Medical Science And Clinical Research \\ IGM Publication \\ An Official Publication of IGM Publication
}

Review Article \& Case

\title{
Dilemma between Tuberculoma \& Multiple NCC with Complicated Case in JLNMCH, Bhagalpur
}

\author{
Authors \\ Dr Abilesh Kumar ${ }^{1}$, Dr Praveen Jadhav ${ }^{2 *}$, Dr Abhishek Tiwari ${ }^{3}$, Dr Pandu Ajmeera ${ }^{4}$ \\ ${ }^{1} \mathrm{MD}$ (Internal Medicine), FRCP, Associate Professor, Postgraduate Medicine Department, JLNMCH, \\ Bhagalpur, Bihar \\ ${ }^{2,3,4}$ MD (Internal Medicine P.G. Student), Postgraduate Medicine Department, JLNMCH, Bhagalpur, Bihar \\ *Corresponding Author \\ Dr Praveen Suresh Jadhav
}

Address: PG Hostel Room No 4, Infront of Mayagang Hospital, Bhagalpur, Bihar, Pin 812001

\begin{abstract}
Aim: To study in details the dilemma between multiple neurocystycercosis (NCC) \& tuberculoma.

Materials \& Methods: Blood, ELISA FOR NCC,CECT SCAN, MRI SCAN,MRS \& Others

Conclusion: Dilemma between tuberculoma and multiple NCC is common in India. Even though there is advancement in medicine and imaging still the uncertainty continues between NCC \& tuberculoma. Our patient finally after so many opinions \& investigation is diagnosed as a case of multiple NCC with Meningitis. CECT scan s/o NCC as most of lesions are resolved and also IgG for NCC is positive. Lumbar puncture/CSF examination suggestive of tubercular meningitis. Fundoscopy showing papilloedema which was in favor of meningitis again. So to conclude it we are pointing towards the association between NCC and tubercular meningitis.

Keywords: NCC: Neurocysticercosis; TBM: Tubercular Meningitis.
\end{abstract}

\section{Background}

Multiple ring-enhancing lesions in the brain have been a topic of debate for differentiating between tuberculoma and neurocysticercosis for long. Spontaneous resolution of the ring-enhancing lesions is considered as a major criterion for diagnosis of neurocysticercosis. We report a rare case of multiple ring-enhancing lesions in the brain, which was initially diagnosed as a case of neurocysticercosis but reconsidered as a case of tuberculoma which finally concluded as a case of NCC with TBM.

\section{Case Details}

35 yrs female ANSHU DEVI immunocompitant (HIV NEG) admitted in JLNMCH, BGP with c/o: headache since 6 month, altered sensorium since 1 day but no convulsions, fever \& meningeal signs was diagnosed as NCC on CT SCAN (P/C) \& treated with albendazole, dexona \& epsolin, relieved \& discharged. 


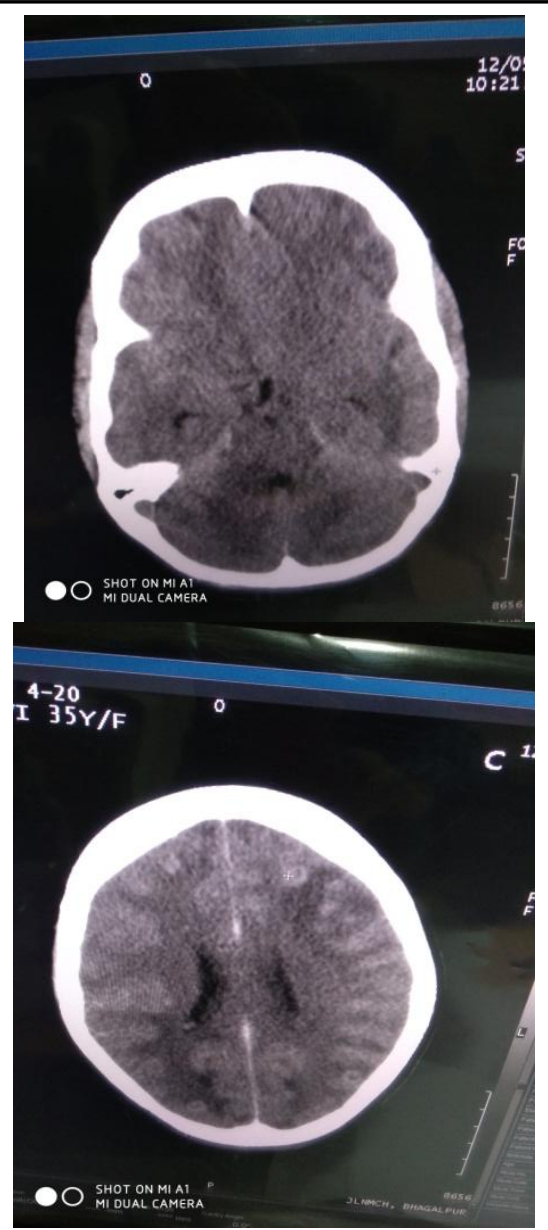

Pt was readmitted $2^{\text {nd }}$ time after 12 days with complains of headache \& vomiting without convulsion, fever and meningeal signs \& was diagnosed as NCC on CTSCAN (P/C) again but when undergone CEMRI it reveals multiple tiny round to oval ring enhancing lesions with mild surrounding edema scattered diffusely in $\mathrm{B} / \mathrm{L}$ cerebral \& cerebellar hemisphere, brainstem, basal ganglia \& thalami showing restriction on DWI images...suggesting infective granuloma (TUBERCULOMA) \& started ATT drugs with dexona and discharge after relieving of symptom.MRS also concluded tuberculoma.
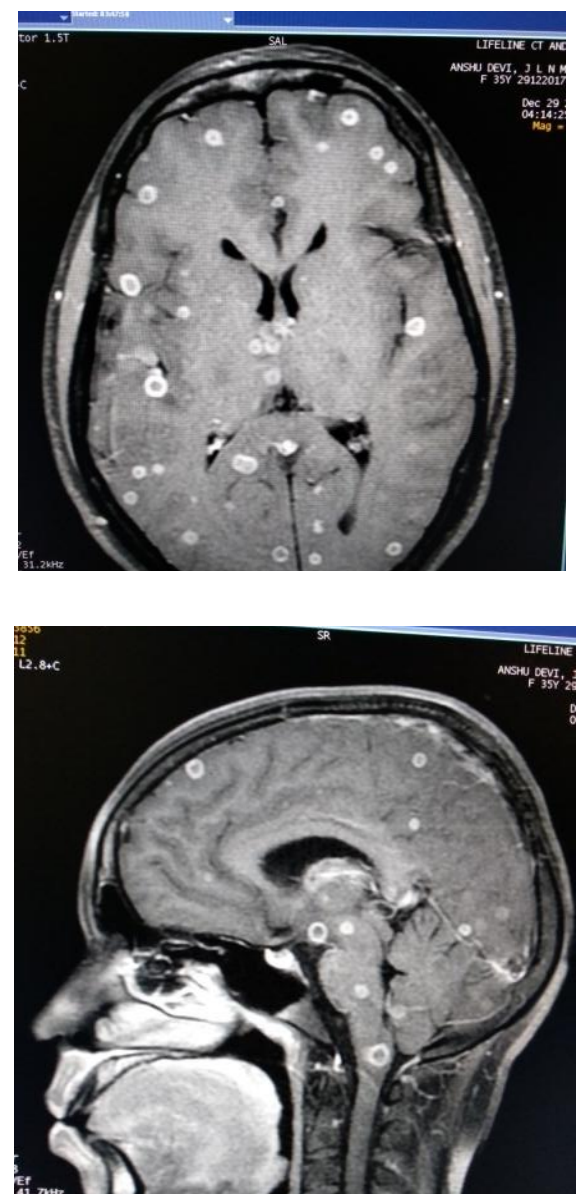

Pt was admitted for 3 rd time with similar c/o headache but this time with convulsions (GTCS) two times/d treatment continued for tuberculoma but after 4 days of treatment pt develop mixed Stevens -johnson \$ \&TEN \$ \& all drug treatment stopped . Pt was treated symptomatically in ICU with anti histaminics, dexona \& topical eye and skin treatment,pt responded to the treatment \& shifted to general ward $\mathrm{f} / \mathrm{b}$ discharged by continuing the treatment of NCC for $6 \mathrm{wks}$ as report of ELISA FOR NCC is positive for IgG.

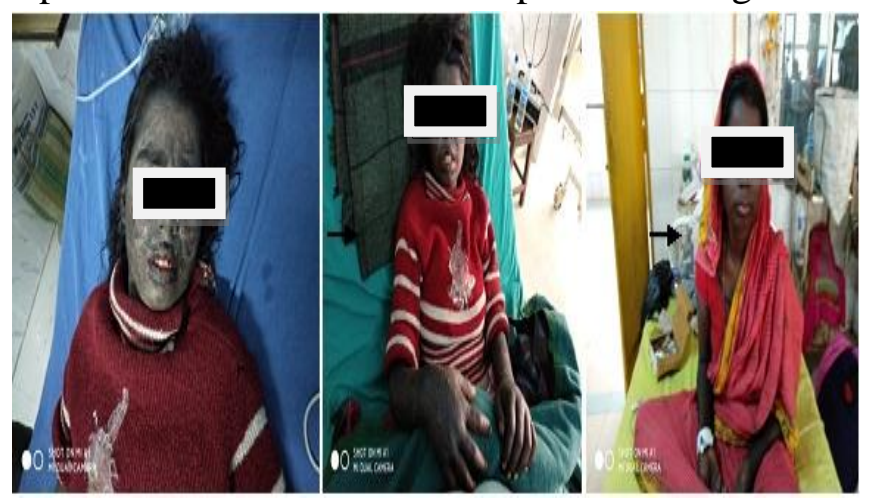

Pt. is now admitted for $4^{\text {th }}$ time after taking treatment for 6 wks of NCC with c/o diminution 
of vision since five days and headache $\mathrm{f} / \mathrm{b}$ convulsions one attack/day not associated with fever but irritable with no other meningeal signs we have undergone lumbar puncture which came out with tubercular meningitis this time and we started treatment ATT with pyrazinamide $1000 \mathrm{mg}$ \& streptomycin $0.75 \mathrm{gm} \mathrm{f} / \mathrm{b}$ rifampicin $450 \mathrm{mg}$ after three days $\mathrm{f} / \mathrm{b}$ levofloxacin $500 \mathrm{mg}$ so we by exclusion made isoniazide as culprit drug for drug allergic reaction which was developed during the previous admission time.we also undergone ophthalmic opinion which came out to be grade 2 papilloedema in both eyes .CECT finding this time suggests the multiple ring enhancing lesions have resolved mostly around $80 \%$ of previous CECT findings
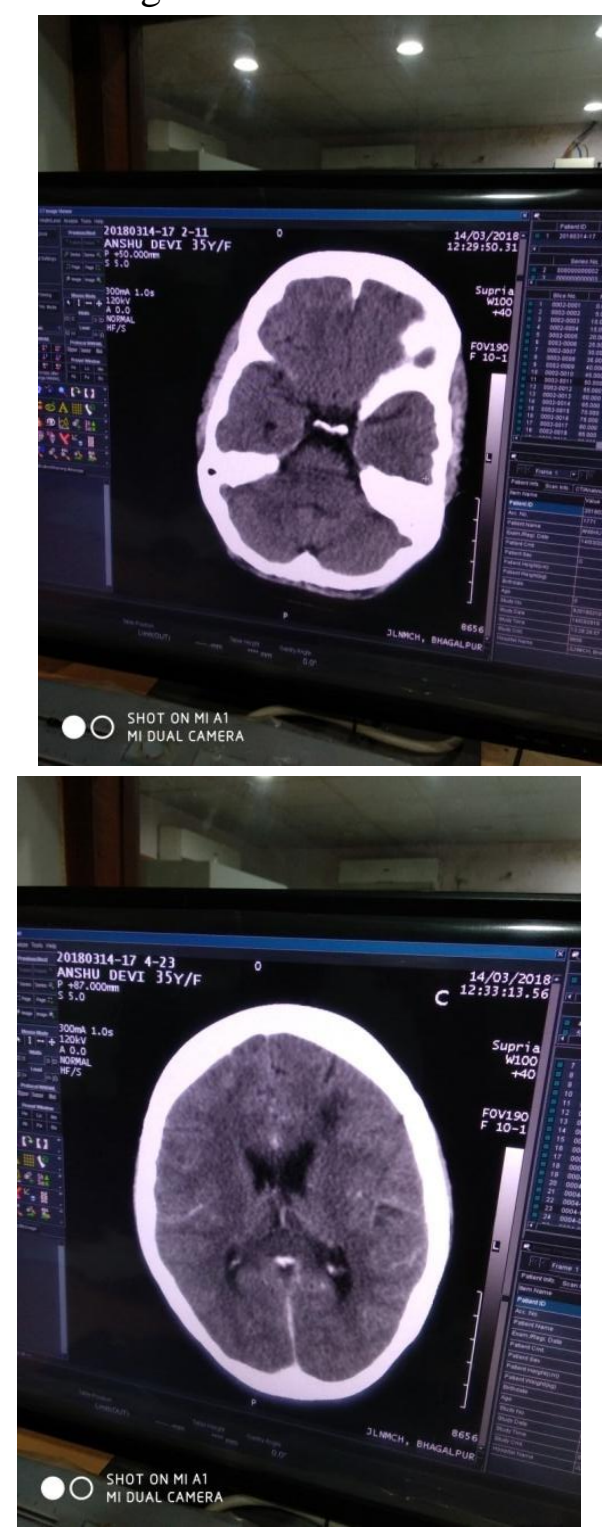

Examination Details: (fourth time admission details)

$\mathrm{G} / \mathrm{E}$ : pt is conscious, cooperative w.o.t. time, place, person. vitals $r$ normal.

(Afebrile \& diminished vision)

S/E: Normal (CVS;RS;P/A;CNS)

FUNDOSCOPY: Papillodema PRESENT

\section{Investigations}

CXR: N; ECG: N; CBC: N ABG: N; RBS: N

LP CSF Finding: TUBERCULAR MENINGITIS

(Physical: COB WEB -PRESENT)

Chemical: GLUCOSE-29.01 mg/dl, PROTEIN$102.60 \mathrm{mg} / \mathrm{dl}, \mathrm{ADA}-10.70 \mathrm{U} / \mathrm{L}$

Microscopic: 378 cells/cumm, mostly lymphocytes $98 \%, \mathrm{RBC} \quad 02 \%$ GRAM \& $\mathrm{ZN}$ STAINED SMEAR -N)

ELISA: IgG positive ( 0.460 OD units) \{positive > 0.3 OD units $\}$

CT SCAN: INFECTIVE GRANULOMA (MULTIPLE NCC)

CEMRI: reveals multiple tiny round to oval shaped ring enhancing lesions with mild surrounding edema, scattered diffusely in b/l cerebral \& cerebellar hemisphere, brainstem basal ganglia \& thalami, showing restriction on DWI images ---s/o INFECTIVE GRANULOMA (TUBERCULOMA)

MRS: reveals mildly elevated choline and LL peak with normal appearing NAA \&Cr peak---s/o INFECTIVE

GRANULOMA

(TUBERCULOMA)

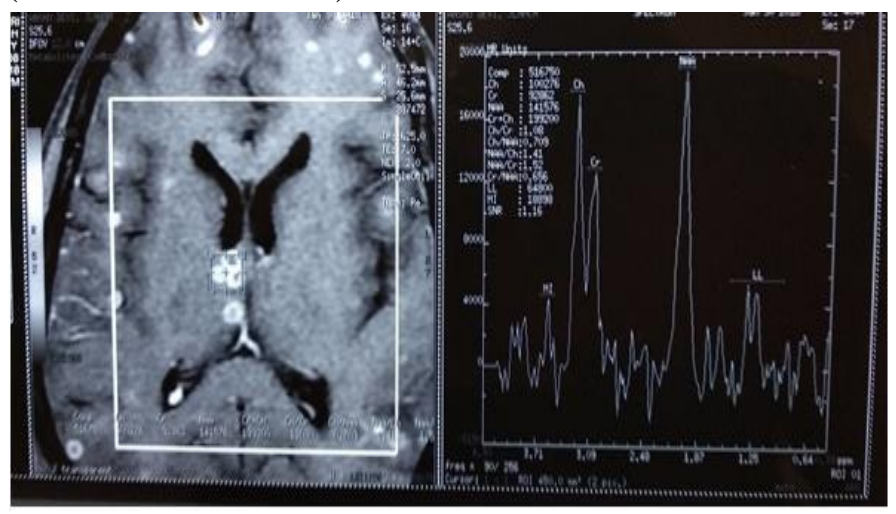

\section{Conclusion}

1) Dilemma between tuberculoma and multiple NCC is common in india 
2) Even though there in advancement in medicine and imaging still the uncertainty continues between NCC \&tuberculoma

3) Patient is finally after so many opinions diagnosed as a case of multiple NCC with Meningitis

4) CECT scan s/o NCC as most of lesions are resolved and also $\mathrm{IGg}$ for $\mathrm{NCC}$ is positive

5) Lumbar puncture/csf examination s/o meningitis

6) Fundoscopy showing papilloedema which was in favor of meningitis

\section{References}

1. Seth R, Kalra V, Sharma U, Jagannathan $\mathrm{N}$. Magnetic resonance spectroscopy in ring enhancing lesions. Indian pediatrics. 2010;47(9):803-4.

2. Rudresh K MKM, Karthik, Sebastin J. Clinical and Aetiological Profile of Ringenhancing Lesions on CT Brain. Journal of Indian Academy of Clinical Medicine. 2008;9 (2):100-2.

3. RK G. Single enhancing computerized tomography- detected lesion in immunocompetent patients. Neurosurg Focus. 2002;12(6):1-9.

4. Singhi P RM. Focal seizure with single small ring enhancing lesion Seminars in Pediatric. Neurology. 1999;6(3):196-201.

5. Sethi PP, Wadia RS, Kiyawat DP, Ichaporia NR, Kothari SS, Sangle SA, et al. Ring or disc enhancing lesions in epilepsy in India. The Journal of tropical medicine and hygiene. 1994;97(6):34753.

6. Jain AP JR, Lathia T. Single Small Enhancing Computed Tomography Lesion: A Review. Journal of Indian Academy of Clinical Medicine. 2005; $6(2): 114-21$.
7. Gujar SK, Maheshwari S, BjorkmanBurtscher I, Sundgren PC. Magnetic resonance spectroscopy. J Neuroophthalmol 2005;25(3):217-26.

8. Pretell EJ MCJ, Garcia HH, Alvarado M, Bustos JA, Martinot C. Differential diagnosis between cerebral tuberculosis and neurocysticercosis by magnetic resonance spectroscopy. J Comput Assist Tomogr. 2005;29:112-4.

9. Santy K, Nan P, Chantana Y, Laurent D, Nadal D, Richner B. The diagnosis of brain tuberculoma by (1) H-magnetic resonance spectroscopy. Eur J Pediatr. 2011;170(3):379-87.

10. Gutch M, Jain N, Agrawal A, Modi A. MR spectroscopy in tuberculoma of brain. BMJ Case Rep. 2012:2012. 